\title{
SIMULTANEOUS EMBEDDINGS OF FINITE DIMENSIONAL DIVISION ALGEBRAS
}

\author{
LOUIS ROWEN AND DAVID SALTMAN
}

(Communicated by Harm Derksen)

\begin{abstract}
L. Small asked whether two finite dimensional division algebras containing a common central subfield $F$ are embeddable in a common division algebra. Although we have a counterexample, the question is answered affirmatively for division algebras whose centers are finitely generated over a common perfect subfield.
\end{abstract}

A celebrated theorem of P.M. Cohn $[\mathrm{C}$ says that for any two division rings (not necessarily finite dimensional) over a field $F$, their amalgamated product over $F$ is a domain which can be embedded in a division ring. Note that even with the two initial division rings being finite dimensional over their centers, the resulting division ring is never finite dimensional over its center. Perhaps this led Lance Small to ask the following question:

We say $D / K$ is a division algebra when $D$ is a division ring finite dimensional over its center $K$. Assume that $K_{1}$ and $K_{2}$ are fields with a given common subfield $F_{0}$. Small asked whether any two division algebras $D_{1} / K_{1}$ and $D_{2} / K_{2}$ can be embedded in some third division algebra $E$.

Let us frame this in several versions, in order of increasing strength:

(1) Is there some $E$ containing both $D_{1}$ and $D_{2}$ ?

(2) Is there some $K$-division algebra $E$ containing both $D_{1}$ and $D_{2}$ in such a way that $K \subset K_{i}$ for $i=1,2$ ?

(3) If $D_{1} / K_{1}$ and $D_{2} / K_{2}$ are division algebras of respective degrees $p^{t_{1}}$ and $p^{t_{2}}$, then can $D_{1} / K_{1}$ and $D_{2} / K_{2}$ be embedded into a single division algebra $E / K$ of degree $p^{t}$, and is there a bound for $t$ in terms of $t_{1}$ and $t_{2}$ ? What would be the best bound?

Question (3) is natural to ask, since every division algebra is a tensor product of division algebras of prime power degree. In this paper, we focus on Question (1). We start with a surprisingly straightforward counterexample in the next section, but then show that a positive solution exists for division algebras finitely generated over a common subfield which is either algebraically closed or the prime subfield (Theorem 2.6).

Received by the editors September 13, 2010 and, in revised form, April 17, 2011.

2010 Mathematics Subject Classification. Primary 16K20, 16K40, 12E15; Secondary 16K50.

Key words and phrases. Division algebra, cyclic algebra, embedding, Schur index, index reduction, Weyl transfer, profinite group.

This work was supported by the U.S.-Israel Binational Science Foundation (grant No. 2010149). 
When $L / F$ is a cyclic Galois extension of dimension $n$ and $a \in F, \Delta(L / F, a) / F$ denotes the $F$-central cyclic algebra having maximal subfield $L$, together with some element $z$ inducing the automorphism generating $\operatorname{Gal}(L / F)$, satisfying $z^{n}=a$. If $F_{1} / F$ and $F_{2} / F$ are field extensions, then an amalgamation of the $F_{i}$ over $F$ is the field of fractions of a quotient of $F_{1} \otimes_{F} F_{2}$. If $F_{1} \otimes_{F} F_{2}$ is itself a domain, then its field of fractions is called the free amalgamation of the $F_{i}$ over $F$. Recall from [J] or [La1, Proposition 4 of $\S X .6]$ that any finitely generated, separably generated field extension is a finite separable extension of a purely transcendental field extension. In particular, this is the case for any finitely generated field extension in characteristic 0 .

\section{A counterexample}

Suppose, first of all, that $D_{1} / K_{1}$ is an f.d. division algebra embedded in the division algebra $E / K$, so $K_{1}$ and $K$ share the same prime subfield $P$. There is a tower of subalgebras $K \subseteq K_{1} K \subset D_{1} K \subset E$, where $K_{1} K$ must be an amalgamation of $K_{1}$ and $K$ over $P$.

More specifically, suppose $p_{1} \neq p_{2}$ are primes. Let $G$ be the infinite cyclic profinite $p_{2}$-group, the inverse limit of all $\mathbb{Z} / p_{2}{ }^{n} \mathbb{Z}$. Take $K_{1} / \mathbb{Q}$ Galois with group $G$. (For example, $K_{1}$ could be contained in the infinite extension of $\mathbb{Q}$ obtained by adjoining all $p_{2}^{n}$ roots of 1 .) Note that $G$ has no finite subgroups. The field extension $K_{1} K / K$ has Galois group a subgroup of $G$ and must be finite dimensional (being inside $E / K)$, and so must be trivial. That is, $K_{1} K=K$, implying $K_{1} \subseteq K$.

Next, take $D_{1}^{\prime} / \mathbb{Q}$ of degree $p_{1}$ and let $D_{1}=D_{1}^{\prime} \otimes_{\mathbb{Q}} K_{1}$, a division algebra since $K_{1} / \mathbb{Q}$ is a pro- $p_{2}$ extension, and let $D_{2} / \mathbb{Q}$ be any division algebra split by $K_{1}$. For example, there is a cyclic degree $p_{2}$ extension $L / \mathbb{Q}$ such that $L \subset K_{1}$. By class field theory (cf. La2, Theorem 4 of $\S X .3]$ ), there is some element of $a \in \mathbb{Q}$ which is not a norm from $L$, so by Wedderburn's criterion, the cyclic algebra $\Delta(L / \mathbb{Q}, a)$ is a degree $p_{2}$ division algebra with maximal subfield $L$.

Proposition 1.1. There is no division algebra $E / K$ containing both $D_{1}$ and $D_{2}$.

Proof. If $E / K$ contains both $D_{1}$ and $D_{2}$, then $K_{1} \subseteq K$ is central, by the above paragraph, so $E$ contains $D_{2} K_{1}$, which is a division algebra but also a homomorphic image of the split algebra $D_{2} \otimes_{\mathbb{Q}} K_{1}$ and thus is commutative, a contradiction.

The rationale for this example is that the centers are incompatible in some sense.

\section{Positive Results}

Now we turn to the positive result, where we observe that the above incompatibility is impossible when the $K_{i}$ are both finitely generated over an algebraically closed or prime field. In fact, we observe that it suffices for the $K_{i}$ both to be finitely generated over a common perfect field (Theorem 2.6).

We approach the problem via [Sa]. First let us fix some notation. All algebras are over a given field $F_{0}$. We write $\operatorname{index}(D)$ for the (Schur) index of a division algebra $D$. Fixing $r>1$, let $F \supset F_{0}$ be a field, and let $U D(F, n) / Z(F, n)$ denote the generic division algebra of degree $n$ over $F$ in $r$ indeterminates. We write $Z$ for $Z(F, n)$. The letter $t$ always denotes an indeterminate. We begin with some lemmas. 
Lemma 2.1. There is a field $K(t) \supset Z$ and a degree $n$ cyclic extension $L / K(t)$ such that $L / F$ is rational and $U D(F, n) \otimes_{Z} K(t)=\Delta(L / K(t), t)$.

Proof. We may assume that $r=2$. Write $Z=F(X \oplus Y)^{S_{n}}$ as usual (see Sa, p. 322). Let $C_{n} \subset S_{n}$ be generated by the $n$ cycle $(1,2, \ldots, n)$. Over $C_{n}, Y \cong M \oplus \mathbb{Z}$ where $M$ is a free $C_{n}$ lattice. We can set $L=F(X \oplus M), K=F(X)^{C_{n}}$ so that $K(t)=L^{C_{n}}$, and take $t$ to be the generator of $\mathbb{Z}$.

Lemma 2.2. Suppose $F$ is a field, $D / F$ is a division algebra, and $b \in \mathbb{N}$. Set

$$
A=D \otimes_{F} U D(F, n)^{b}=\left(D \otimes_{F} Z\right) \otimes_{Z} U D(F, n)^{b} .
$$

Then $\operatorname{index}(A)$ is the degree of $D$ times $n /(n, b)=\operatorname{index}\left(U D(F, n)^{b}\right)$.

Proof. Since $U D(F, n)$ has index equal exponent, the index of any power is equal to the exponent. In fact, if $b=b^{\prime}(n, b)$ and $n=n^{\prime}(n, b)$, then $\left(b^{\prime}, n^{\prime}\right)=1$. Thus $U D(F, n)^{b}=\left(U D(F, n)^{(n, b)}\right)^{b^{\prime}}$ and the index and exponent of $U D(F, n)^{b}$ is the same as that of $\left(U D(F, n)^{(n, b)}\right)$. That is, we may assume $b \mid n$.

By Lemma 2.1, there is a field $K(t) \supset Z$ and a degree $n$ cyclic extension $L / K(t)$ such that $D \otimes_{F} L$ is a division algebra and $U D(F, n) \otimes_{Z} K(t)=\Delta(L / K(t), t)$. Of course, by Galois theory, $\Delta(L / K(t), t)^{b}$ is equal in the Brauer group to $\Delta\left(L^{\prime} / K(t), t\right)$ where $L / L^{\prime}$ has dimension $b$. We conclude by showing that $\left(D \otimes_{F} K(t)\right) \otimes_{K(t)}$ $\Delta\left(L^{\prime} / K(t), t\right)$ is a division algebra. Indeed, taking $y \in \Delta\left(L^{\prime} / K(t), t\right)$ such that $y^{n / b}=t$ and $y x y^{-1}=\sigma(x)$ for all $x \in L^{\prime}$, where $\sigma$ generates the Galois group of $L^{\prime}$ over $K(t)$, we note that the skew polynomial algebra $D[y ; \sigma]$ satisfies a polynomial identity, so it has a division algebra of fractions obtained by inverting the central elements, and this is precisely $\left(D \otimes_{F} K(t)\right) \otimes_{K(t)} \Delta\left(L^{\prime} / K(t), t\right)$.

We are in the game of embedding division algebras into bigger division algebras. The key method is the following.

Theorem 2.3. Suppose $D / K$ is a division algebra of degree a and $K / F$ is a field extension of dimension $b$. Assume $E / F$ is a division algebra of degree $N=$ nab. Then $D$ is isomorphic to a subalgebra of $E$ over $F$ if and only if $\left(E \otimes_{F} K\right) \otimes_{K} D^{o p}$ has (Schur) index dividing $n$. Furthermore, if this index divides $n$, then it is equal to $n$.

Proof. Suppose $D \subset E$. In particular, $K \subset E$ and so $E \otimes_{F} K$ has index $N / b$, and we set $E^{\prime} / K$ to be the associated division algebra which is the centralizer of $K$ in $E$. Then $D \subset E^{\prime}$ and we take $D^{\prime}$ to be its centralizer, implying $E^{\prime}=D \otimes_{K} D^{\prime}$. Since $D^{\prime}$ has degree $n$, we have proven one direction.

Conversely, suppose index $\left(\left(E \otimes_{F} K\right) \otimes_{K} D^{o p}\right)$ divides $n$. Then $\operatorname{index}\left(E \otimes_{F} K\right)$ divides $n a$, implying index $\left(E \otimes_{F} K\right)=n a$ since $\operatorname{index}\left(E \otimes_{F} K\right) \geq \frac{N}{b}=n a$. Thus, $\left(E \otimes_{F} K\right) \otimes_{K} D^{o p} \sim A$, where $A / K$ has degree $n$, implying $\left[E \otimes_{F} K\right]$ is equal in the Brauer group to $[D][A]$.

Let $E^{\prime}$ be the centralizer of $K$ in $E$. Since the degrees agree, $E^{\prime} \cong D \otimes_{K} A$.

We are going to force one algebra inside another by using partial splitting fields and Weil transfers. More specifically, let $A / K$ be a central simple algebra and $n$ be an integer dividing the degree of $A / K$. Let $V_{n}(A)$ be the variety of rank $n$ left ideals of $A$ and let $K_{n}(A)$ be its field of fractions. Then for any field $K^{\prime} \supset K$, $V_{n}(A)$ has a $K^{\prime}$ point if and only if $A \otimes_{K} K^{\prime}$ has index dividing $n$. 
Next we set $W_{n}(A)$ to be the Weil transfer to $F$ of $V_{n}(A)$, so for $F^{\prime} \supset F, W_{n}(A)$ has an $F^{\prime}$ point if and only if $V_{n}(A)$ has a $K \otimes_{F} F^{\prime}$ point (and in fact there is a natural correspondence). Let $F_{n}(A)$ denote the field of rational functions of $W_{n}(A)$. Then index $\left(A \otimes_{F} K F_{n}(A)\right)$ divides $n$.

The important tool for using this construction is the following result ([Sa] $)$ about index reduction, for which we need to introduce more notation. Let $K / F$ be finite separable with Galois closure $\bar{K} / F$. Let $G$ be the Galois group of $\bar{K} / F$ and $H \subset G$ be the subgroup corresponding to $\bar{K} / K$. If $r$ is the degree of $A / K$, then we can define an "action" of the $G$ module $R=(\mathbb{Z} / r \mathbb{Z})[G / H]$ as follows. Let $\bar{A}=A \otimes_{K} \bar{K}$, so $H$ has a natural semilinear action on $\bar{A}$ and for any $g \in G$ we can define the $g$ twist $g(\bar{A})$. Of course, for $g \in G, g H g^{-1}$ has a natural semilinear action on $g(\bar{A})$.

For $\alpha \in R$, define $H_{\alpha}=\{g \in G \mid g \alpha=\alpha\}$. Define $K(\alpha)=\bar{K}^{H_{\alpha}}$. Write

$$
\alpha=\sum n_{g H} g H
$$

then the $n_{g H}$ are constant on $H_{\alpha}$-orbits. Fix a coset $g H$ and set $e=n_{g H}$. Let $L \subset H_{\alpha}$ be the stabilizer of $g H$. Let $\mathcal{O}=\left\{g_{i} H\right\}$ be the orbit of $H_{\alpha}$ containing $g H$ so $e=n_{g_{i} H}$ for all $i$. Then $L$ acts naturally on $g(\bar{A})$ and $H_{\alpha}$ acts on $B_{g H}$, which is the tensor product over $\bar{K}$ of $g_{i}(\bar{A})^{e}$, one for each $g_{i} H$ in $\mathcal{O}$.

Now we let $g H$ vary, one for each $H_{\alpha}$ orbit. Tensor over $\bar{K}$ all the $B_{g H}$ defined above and call the resulting $\bar{K}$ algebra $B$. Note that $\bar{K}$ is the center of $B$. Define $A^{\alpha}$ to be the $H_{\alpha}$ invariant subring of $B$. Then $A^{\alpha}$ has center $K(\alpha)$.

Finally, for $\alpha=\sum n_{g H} g H$ as above, define

$$
|\alpha|=\prod_{g H} \frac{n}{\left(n, n_{g H}\right)} .
$$

Theorem 2.4 ([Sa], p. 332). With notation as above, suppose $B / F$ is any central simple algebra (over $F$ ). Then the index of $B \otimes_{F} F_{n}(A)$ is the gcd of all the integers

$$
\text { index }\left(B \otimes_{F} A^{\alpha}\right)[K(\alpha): F]|\alpha| \text {, }
$$

taken over all $\alpha \in R$.

We actually need a double version of the above result. Let us assume that $K / F$ and $K^{\prime} / F$ are finite separable with Galois closures $\bar{K} / F$ and $\bar{K}^{\prime} / F$ and corresponding groups $G \supset H$ and $G^{\prime} \supset H^{\prime}$. For convenience we may assume that $\bar{K} / F$ and $\bar{K}^{\prime} / F$ are linearly disjoint. Let $A / K$ and $A^{\prime} / K^{\prime}$ be central simple algebras and let $F_{n, n^{\prime}}\left(A, A^{\prime}\right)$ denote the free amalgamation of the fields $F_{n}(A)$ and $F_{n^{\prime}}\left(A^{\prime}\right)$ over $F$. If $A^{\prime} / K^{\prime}$ has degree $r^{\prime}$, set $R^{\prime}=\left(\mathbb{Z} / r^{\prime} \mathbb{Z}\right)\left[G^{\prime} / H^{\prime}\right]$ as above. If $\alpha \in R$ and $\beta \in R^{\prime}$, set $K(\alpha, \beta)=K(\alpha) \otimes_{F} K^{\prime}(\beta)$. Finally, write $\beta=\sum_{g H^{\prime}} m_{g H^{\prime}} g H^{\prime} \in R^{\prime}$ and set

$$
|\beta|=\prod_{g H^{\prime}} \frac{n^{\prime}}{\left(n^{\prime}, m_{g H^{\prime}}\right)} .
$$

Theorem 2.5. Suppose $B / F$ is a central simple algebra and set

$$
B(\alpha, \beta)=B \otimes_{F} K(\alpha, \beta) .
$$

Then the (Schur) index $\mathbf{i}:=\operatorname{index}\left(B \otimes_{F} F_{n, n^{\prime}}\left(A, A^{\prime}\right)\right)$ is the gcd of the integers

$$
\begin{array}{r}
\operatorname{index}\left(\left(B(\alpha, \beta) \otimes_{K(\alpha, \beta)}\left(A^{\alpha} \otimes_{K(\alpha)} K(\alpha, \beta)\right) \otimes_{K(\alpha, \beta)}\left(A^{\prime \beta} \otimes_{K^{\prime}(\beta)} K(\alpha, \beta)\right)\right)\right. \\
\times[K(\alpha, \beta): F]|\alpha \| \beta|,
\end{array}
$$

ranging over all $\alpha \in R$ and $\beta \in R^{\prime}$. 
Proof. The basic idea here is to apply Theorem 2.4 twice, noting that $[K(\alpha, \beta)$ : $F]=[K(\alpha): F]\left[K^{\prime}(\beta): F\right]$. Put $B^{\prime}=B \otimes_{F} F_{n}(A)$. Then, by Theorem [2.4 using the fact that $\left[F_{n} K^{\prime}(\beta): F_{n}(A)\right]=\left[K^{\prime}(\beta): F\right]$, $\mathbf{i}$ is the gcd of all integers

$$
\text { index }\left(\left(B^{\prime} \otimes_{F_{n}(A)} F_{n} K^{\prime}(\beta)\right) \otimes_{F_{n} K^{\prime}(\beta)}\left(A^{\prime \beta} \otimes_{K^{\prime}(\beta)} F_{n} K^{\prime}(\beta)\right)\right)\left[K^{\prime}(\beta): F\right]|\beta|,
$$

where $F_{n} K^{\prime}(\beta)$ is the join of $F_{n}(A)$ and $K^{\prime}(\beta)$ over $F$. Note that $F_{n} K^{\prime}(\beta)$ is the function field of $W_{n}\left(A \otimes_{F} K(\beta)\right)$, which is the $K(\alpha, \beta) / K^{\prime}(\beta)$ transfer of $V_{n}\left(A \otimes_{F}\right.$ $\left.K^{\prime}(\beta)\right)$. Now by Theorem 2.4 again, using $B^{\prime}$ instead of $B$, each

$$
\text { index }\left(\left(B^{\prime} \otimes_{F_{n}(A)} F_{n} K^{\prime}(\beta)\right) \otimes_{F_{n} K^{\prime}(\beta)}\left(A^{\prime \beta} \otimes_{K^{\prime}(\beta)} F_{n} K^{\prime}(\beta)\right)\right)
$$

is the gcd of

$$
\operatorname{index}\left(\left(B \otimes_{F} A^{\alpha}\right) \otimes_{K(\alpha)}\left(A^{\prime \beta} \otimes_{F_{n} K^{\prime}(\beta)} K(\alpha, \beta)\right)\right)\left[K(\alpha, \beta): K^{\prime}(\beta)\right]|\alpha|,
$$

and the result follows.

We are ready for a result generalizing [FSS, Theorem 2.8], which considers the problem of embedding a $K_{1}$-division algebra into a $K$-division algebra, where $K_{1}$ is a finite dimensional field extension of $K$. Now suppose we already know that $D_{1} / K_{1}$ and $D_{2} / K_{2}$ are division algebras of degrees $d_{i}$ and $K_{i} / F$ is separable of degree $e_{i}$. Set $m_{i}=d_{i} e_{i}$ and let $N$ be any multiple of the lcm of $m_{1}^{2}$ and $m_{2}^{2}$.

Theorem 2.6. There is a finitely generated field extension $F^{\prime} \supset F$ such that $F^{\prime} / F$ is separably generated and $F$ is algebraically closed in $F^{\prime}$, together with a division algebra $E^{\prime} / F^{\prime}$ of degree $N$ such that $D_{i} \subset E^{\prime}$ is compatible with $F \subset F^{\prime}$ for $i=1,2$.

Proof. Set $n_{i}=N / m_{i}$, noting that $n_{i}$ is a multiple of $m_{i}$. Set

$$
E=U D(F, N)
$$

with center $Z$. We will extend $Z$ so that the $D_{i}$ embed in the base extension of $E$. To achieve this we set $F^{\prime}=F_{n_{1}, n_{2}}\left(A_{1}, A_{2}\right)$ where

$$
A_{i}=\left(D_{i}^{\circ} \otimes_{K_{i}} K_{i} Z\right) \otimes_{K_{i} Z}\left(E \otimes_{Z} K_{i} Z\right) .
$$

Note that $\left(E \otimes_{Z} K_{i} Z\right)$ is just the generic division algebra over $K_{i}$. Also note that $E^{\prime}=E \otimes_{Z} F^{\prime}$ has both $D_{i}$ embedded, by Theorem 2.3 , because, in view of Lemma 2.2. we have suitably reduced the index of both $A_{i}$. The problem is to show that $E^{\prime}$ is a division algebra, i.e., that index $\left(E^{\prime}\right)=N$, and for this we apply Theorem 2.5. In applying this theorem, note that the degree of $A_{1}$ is $N d_{1}$, and so is a multiple of $n_{1}$. We make a similar comment about the degree of $A_{2}$.

To apply Theorem 2.5, we need to get a handle on index $\left(\left(E \otimes_{Z} Z K(\alpha, \beta)\right)^{1+\alpha+\beta} \otimes\left(D_{1}^{-\alpha} \otimes_{K(\alpha)} Z K(\alpha, \beta)\right) \otimes\left(D_{2}^{-\beta} \otimes_{K^{\prime}(\beta)} Z K(\alpha, \beta)\right)\right)$, where the unsubscripted tensors are over $Z K(\alpha, \beta)$. Write $\alpha=\sum n_{g H} g H$ and set $a=\sum n_{g H}$ and similarly for $\beta$ and $b$. Note that $E$ is not moved by either Galois group, so $\left(E \otimes_{Z} K(\alpha, \beta) Z\right)^{1+\alpha+\beta}$ is $E^{1+a+b} \otimes_{Z} K(\alpha, \beta) Z$, which has index $N /(N, 1+a+b)$, which we define to be $N_{a, b}$. By Lemma 2.2 the above index is

$$
\text { index }\left(\left(D_{1}^{-\alpha} \otimes_{K(\alpha)} K(\alpha, \beta)\right)\right) \otimes_{K(\alpha, \beta)}\left(D_{2}^{-\beta} \otimes_{K^{\prime}(\beta)} K(\alpha, \beta)\right) N_{a, b},
$$


and so we want to show that $N$ divides the expression

(2)

$$
\text { index }\left(\left(D_{1}^{\alpha} \otimes_{K(\alpha)} K(\alpha, \beta)\right) \otimes_{K(\alpha, \beta)}\left(D_{2}^{\beta} \otimes_{K^{\prime}(\beta)} K(\alpha, \beta)\right)[K(\alpha, \beta): F]|\alpha||\beta| N_{a, b}\right. \text {. }
$$

We show the needed divisibility prime by prime. So assume for $p$ prime that $p^{s}$ divides $N$ exactly, in the sense that $\frac{N}{p^{s}}$ is prime to $p$. Likewise, assume that $p^{t_{i}}$ divides $n_{i}$ exactly. Since $N=n_{i} d_{i} e_{i}$ and $n_{i}$ is a multiple of $d_{i} e_{i}$, we have $2 t_{i} \geq s$ and also $t_{1}+t_{2} \geq s$. If $1+a+b$ is prime to $p$ we are done. Thus we assume $p$ divides

$$
1+\sum_{g H} n_{g H}+\sum_{g H^{\prime}} m_{g H^{\prime}}
$$

and this implies that at least one summand in

$$
\sum_{g H} n_{g h}+\sum_{g H^{\prime}} m_{g H^{\prime}}
$$

is prime to $p$. If any term in (4), say $n_{g H}$, is prime to $p$, then $\frac{n}{\left(n, n_{g H}\right)}$ is divisible by $p^{t_{1}}$. Thus, if two terms in (4) are prime to $p$, then $p^{2 t_{1}}$ or $p^{t_{1}+t_{2}}$ or $p^{2 t_{2}}$ divides $|\alpha||\beta|$, and again we are done.

Thus we assume that $p$ is prime to exactly one summand in (44). Replacing $\alpha$ by $g^{-1} \alpha$, we assume that only $n_{H}$ is prime to $p$. It follows that $H_{\alpha}$ fixes the trivial coset $H$ and so $H_{\alpha} \subseteq H$, implying $K(\alpha) \supseteq K$. Set $\alpha^{\prime}=\alpha-n_{H} H$; thus,

$$
\left|\alpha^{\prime}\right|=\prod_{g H \neq H} \frac{n}{\left(n, n_{g H}\right)} .
$$

We know that $K(\alpha)=[K(\alpha): K][K: F]$. Write $s=s_{1}+s_{2}+s_{3}$ where $p^{s_{1}}$ is the exact power of $p$ dividing $n, p^{s_{2}}$ is the exact power dividing $d_{1}$, and $p^{s_{3}}$ is the exact power dividing $e_{1}$. Note that $p^{s_{1}}$ divides $\frac{n}{\left(n, n_{H}\right)}$, and of course $p^{s_{3}}$ divides $[K: F]$. Thus it suffices to show that $p^{s_{2}}$ divides

$$
\operatorname{index}\left(D^{\prime \prime}\right)[K(\alpha, \beta): K]\left|\alpha^{\prime}\right||\beta|,
$$

where

$$
D^{\prime \prime}=\left(D_{1}^{\alpha} \otimes_{K(\alpha)} K(\alpha, \beta)\right) \otimes_{K(\alpha, \beta)}\left(D_{2}^{\beta} \otimes_{K^{\prime}(\beta)} K(\alpha, \beta)\right) .
$$

We will prove in fact that (5) is divisible by $d_{1}$. Note that

$$
\left(D_{1}^{\alpha} \otimes_{K(\alpha)} K(\alpha, \beta)\right)=\left(D_{1} \otimes_{K} K(\alpha, \beta)\right) \otimes_{K(\alpha, \beta)}\left(D_{1}^{\alpha^{\prime}} \otimes_{K(\alpha)} K(\alpha, \beta)\right) .
$$

We need to estimate some indices. Of course $D_{1}$ has index $d_{1}$, and so over $\bar{K} \bar{K}^{\prime}$, $g\left(D_{1}\right)^{n_{g H}}$ has index dividing $\frac{d_{1}}{\left(d_{1}, n_{g H}\right)}$. Then $D_{1}^{\alpha^{\prime}} \otimes_{K(\alpha)} K(\alpha, \beta)$ has index dividing $\prod_{g H \neq H} \frac{d_{1}}{\left(d_{1}, n_{g H}\right)}$. Similarly $D_{2}^{\beta} \otimes_{K^{\prime}(\beta)} K(\alpha, \beta)$ has index dividing $\prod_{g H^{\prime}} \frac{d_{2}}{\left(d_{2}, m_{g H^{\prime}}\right)}$.

We need a trivial lemma.

Lemma 2.7. Suppose a divides $b$. Then $a /(a, d)$ divides $b /(b, d)$.

Proof. For any prime $p$, the power of $p$ dividing $\frac{a}{(a, d)}$ is less than or equal to the power of $p$ dividing $\frac{b}{(b, d)}$. 
Let $p^{u_{1}}$ be the exact power of $p$ dividing index $\left(D_{1} \otimes_{K} K(\alpha, \beta)\right)$ and let

$$
D^{\#}=\left(D_{1}^{\alpha^{\prime}} \otimes_{K(\alpha)} K(\alpha, \beta)\right) \otimes_{K(\alpha, \beta)}\left(D_{2}^{\beta} \otimes_{K^{\prime}(\beta)} K(\alpha, \beta)\right) .
$$

Also let $p^{u_{2}}$ be the exact power of $p$ dividing index $\left(D^{\#}\right)$. It follows from Lemma 2.7 that $p^{u_{2}}$ divides $\left|\alpha^{\prime} \| \beta\right|$. Also, $D^{\prime \prime}=\left(D_{1}^{n_{H}} \otimes_{K} K(\alpha, \beta)\right) \otimes_{K(\alpha, \beta)} D^{\#}$. Now let $p^{u_{3}}$ be the exact power of $p$ dividing

$$
\operatorname{index}\left(D_{1}^{n_{H}} \otimes_{K} K(\alpha, \beta)\right) .
$$

This is the same as the exact power of $p$ dividing

$$
\operatorname{index}\left(D_{1} \otimes_{K} K(\alpha, \beta)\right) \text {. }
$$

Set $p^{u_{4}}$ to be the exact power of $p$ dividing $[K(\alpha, \beta): K]$. Then $\operatorname{index}\left(D^{\prime \prime}\right)$ is a multiple of $p^{u_{1}-u_{2}}$. Thus index $\left(D^{\prime \prime}\right)\left|\alpha^{\prime}\right||\beta|$ is a multiple of $p^{u_{1}}$ and

$$
\operatorname{index}\left(D^{\prime \prime}\right)\left|\alpha^{\prime}\right||\beta|[K(\alpha, \beta): K]
$$

is a multiple of $p^{u_{1}+u_{4}}$ which is the exact power of $p$ dividing

$$
\operatorname{index}\left(D_{1} \otimes_{K} K(\alpha, \beta)\right)[K(\alpha, \beta): K],
$$

a multiple of $d_{1}$. This proves Theorem 2.6

Theorem 2.8. Suppose $D_{i} / K_{i}$ are division algebras with the $K_{i}$ finite separable extensions of a purely transcendental field extension of a field $F_{0}$. Then the $D_{i}$ can be embedded into a common division algebra $E$ finite over its center.

Proof. Tensoring the base fields by purely transcendental extensions still will leave the $D_{i}$ division algebras, so we may assume that the $K_{i}$ are finite separable extensions of a common subfield purely transcendental over $F_{0}$. Thus, we may apply Theorem 2.6. where here $D_{i}$ takes the role of $D_{i}^{\prime}$ and $E$ takes the role of $E^{\prime}$. (2):

The referee has pointed out that the same techniques can be applied to Question

Theorem 2.9. Suppose $D_{i} / K_{i}$ are division algebras with the $K_{i}$ stably isomorphic or there is a field unirational over both $K_{1}$ and $K_{2}$. Then the $D_{i}$ can be embedded into a common division algebra $E$ finite over its center $K$, such that $K_{i} \subseteq K$ for $i=1,2$.

Proof. Tensoring the base fields by purely transcendental extensions provides them with the same center, to which we can apply Theorem 2.6.

The question remains: What is the lowest possible bound for $\operatorname{deg} E$ ?

\section{ACKNOWLEDGEMENT}

The authors thank the referee for many helpful suggestions, both in style and content. 


\section{REFERENCES}

[C] Cohn, P.M. The embedding of firs in skewfields, Proc. London Math. Soc. (3) 23 (1971), 193-213. MR0297814(45:6866)

[FSS] Fein, B., Saltman, D., and Schacher, M. Embedding problems for finite dimensional division algebras, J. Algebra 167 (1994), 588-626. MR.1287062 (95h:16021)

[J] Jacobson, N., Basic Algebra II, Freeman, 1980. MR.571884 (81g:00001)

[La1] Lang, S. Algebra, Addison-Wesley, 1965. MR0197234(33:5416)

[La2] Lang, S. Algebraic Number Theory, Second edition, Springer Graduate Texts in Mathematics, 110, Springer-Verlag, 1994. MR1282723 (95f:11085)

[Sa] Saltman, D., The Schur index and Moody's theorem, K-Theory 7 (1993), 309-332. MR:1246280(94k:16049)

Department of Mathematics, Bar-Ilan University, Ramat-Gan 52900, Israel

E-mail address: rowen@macs.biu.ac.il

Center for Communications Research-Princeton, 805 Bunn Drive, Princeton, New JerSEY 08540

E-mail address: saltman@idaccr.org 